\title{
Guillain-Barré Syndrome after H1N1 Vaccination in the United States: A Report Using the CDC/FDA Vaccine Adverse Event Reporting System (2009)
}

\author{
Nizar Souayaha Hussam A. Yacouba Hafiz M.R. Khan ${ }^{d}$ P.A. Michas-Martin ${ }^{c}$ \\ Daniel L. Menkes ${ }^{e}$ Leila Maybodi ${ }^{a}$ Adnan I. Qureshi ${ }^{b}$ \\ ${ }^{a}$ Department of Neurology, University of Medicine and Dentistry of New Jersey, Newark, N.J., ${ }^{\text {b } Z e e n a t ~ Q u r e s h i ~}$ \\ Stroke Research Center, University of Minnesota, Minneapolis, Minn., 'New York Medical College, Valhalla, N.Y., \\ ${ }^{\mathrm{d}}$ Department of Biostatistics, Robert Stempel College of Public Health and Social Work, Florida International \\ University, Miami, Fla., and ' Department of Neurology, University of Connecticut Health Center, Farmington, \\ Conn., USA
}

\section{Key Words}

H1N1 influenza • Vaccine • Guillain-Barré syndrome •

Adverse events $\cdot$ Seasonal influenza $\cdot$ Vaccination the first 6 weeks after H1N1 vaccination suggests that some GBS cases may be triggered by H1N1 vaccination. This warrants early recognition, treatment, and active surveillance in the postvaccination setting.

Copyright $\odot 2012$ S. Karger AG, Basel

\begin{abstract}
Background: Although the Guillain-Barré syndrome (GBS) can be associated with the seasonal influenza vaccine, there is no definite evidence that GBS is associated with $\mathrm{H} 1 \mathrm{~N} 1$ influenza vaccination. The objective of this report is to study the occurrence and characteristics of GBS after $\mathrm{H} 1 \mathrm{~N} 1$ vaccine administration in the United States in 2009. Methods: Data were acquired from the Vaccine Adverse Event Reporting System and supplemented by additional information obtained from the Center for Biologics Evaluation and Research, under the Federal Freedom of Information Act. Results: A total of 62 individuals (mean age $46.51 \pm 22.41$ years), 33 of whom were men, developed GBS associated with the H1N1 influenza vaccination in 2009. Sixty GBS cases were reported within 6 weeks after vaccination, with 31 cases $(50.0 \%)$ reported in the first 2 weeks. The estimated rate of occurrence of GBS was 6.2 cases per 10 million vaccinations, which is comparable to the rate of GBS in the general population. Conclusion: The higher rate of GBS reports in
\end{abstract}

\section{Introduction}

The World Health Organization reported that more than 213 countries had laboratory-confirmed cases of H1N1 influenza, which resulted in at least 16,455 deaths, from April 2009 through February 2010 [1]. Notably, in April 2009, the first cases of H1N1 influenza were reported in the United States and Mexico $[2,3]$. In the United States, approximately 61 million subjects were reported to have been infected with the H1N1 influenza between April 2009 and April 2010, resulting in 274,000 H1N1-related hospitalizations and 12,470 deaths [4]. Two forms of vaccine were approved and introduced in the United States in 2009: a monovalent, inactivated $\mathrm{H} 1 \mathrm{~N} 1$ virus and an attenuated $\mathrm{H} 1 \mathrm{~N} 1$ nasal spray. On August 28, 2009, the Centers for Disease Control and Prevention (CDC) Advisory Committee on Immunization

\section{KARGER \\ Fax +41613061234 \\ E-Mail karger@karger.ch}

www.karger.com (c) 2012 S. Karger AG, Basel

$0251-5350 / 12 / 0384-0227 \$ 38.00 / 0$

Accessible online at:

www.karger.com/ned
Nizar Souayah, MD

Department of Neurology, University of Medicine and Dentistry of New Jersey 90 Bergen Street, DOC 8100

Newark NJ, 07103 (USA)

Tel. +1973972 8577, E-Mail souayani@umdnj.edu 
Practices stated that certain groups of subjects who are at highest risk for the influenza infection or infectionrelated complications should be the initial vaccine recipients [5]. Similar guidelines were proposed by other countries depending on their resources and priorities [6]. Several clinical trials showed that the H1N1 influenza vaccine was safe and immunogenic in healthy children, adults, and elderly patients $[7,8]$. Although Guillain-Barré syndrome (GBS) can be associated with the influenza vaccine [9-15], the risk of developing GBS following H1N1 influenza vaccination is unknown. A better understanding of this issue may help in the implementation of the Advisory Committee on Immunization Practices recommendations for $\mathrm{H} 1 \mathrm{~N} 1$ vaccine administration. This study attempted to determine the reporting rate and characteristics of GBS occurrence after administration of the H1N1 vaccine in the United States in 2009, and to compare with GBS occurrence observed after seasonal influenza vaccination.

\section{Methods}

Data were retrieved from the Vaccine Adverse Event Reporting System (VAERS), supplemented by data provided by the Center for Biologics Evaluation and Research, under the Federal Freedom of Information Act. The latter is 1 of 6 main centers for the US Food and Drug Administration, which is a part of the US Department of Health and Human Services. The Center for Biologics Evaluation and Research is responsible for assuring the safety, purity, potency, and effectiveness of biologics (such as vaccines, blood products, and monoclonal antibodies) and related products. The VAERS is a national cooperative program instituted by the CDC and the Food and Drug Administration. The VAERS collects information about adverse events that occur after the administration of licensed vaccines in the United States. This database records the demographic and clinical characteristics of the events, results of diagnostic tests, and patients' outcomes using a nonstandardized format. This database was queried for the calendar year 2009 using the following key words and subject terms: 'Guillain-Barré syndrome', 'acute polyneuropathy', 'Miller-Fisher syndrome', 'paraparesis', 'paraplegia', 'paralysis', 'flaccid paralysis', 'weakness', and 'numbness'. The clinical findings and diagnostic test results were reviewed by a board-certified neuromuscular specialist to identify events that met diagnostic criteria for GBS, as defined by progressive upper and lower extremity weakness with areflexia. The diagnosis was further supported by the presence of an elevated cerebrospinal fluid protein concentration (>45 mg/dl), with fewer than 10 cells $/ \mathrm{mm}^{2}$, and/or electrodiagnostic study findings consistent with primary demyelination. Cases diagnosed as Miller-Fisher syndrome, a variant of GBS, were also included in the study. The occurrence of GBS within 6 weeks of vaccination was considered suggestive of a causal association [13-15]. This was based on the fact that in previous controlled studies that showed an increased risk of GBS after vaccination, most of the risk was within 6 weeks after vaccination [13-15].
The estimated occurrence rate of GBS identified after vaccination with the H1N1 influenza vaccine was compared to that reported after vaccination with the seasonal influenza vaccine.

All cases of GBS occurring within 2 days after vaccination were excluded, as these were unlikely to represent cases with a causal relationship to the $\mathrm{H} 1 \mathrm{~N} 1$ vaccination. The crude reporting rate of GBS occurrence was estimated by dividing the number of events reported within 6 weeks as a fraction of the total number of $\mathrm{H} 1 \mathrm{~N} 1$ vaccinations administered each year. Data for the first 2 weeks after H1N1 influenza vaccination were considered in order to determine the time of peak GBS incidence. This methodology was based on the observation in previous studies that, within the first 6 weeks after vaccination, the highest rate of GBS events occurred within the first 2 weeks after seasonal influenza vaccine administration [13-16]. The total number of vaccinations administered each year was derived from estimates provided by either the CDC or the National Health Interview Survey. The reporting rates and distribution of GBS over time, following $\mathrm{H} 1 \mathrm{~N} 1$ vaccination, were compared with all non-GBS adverse events in 2009.

Data from the Nationwide Inpatient Sample, sponsored by the Agency for Healthcare Research and Quality [17, 18], were used to compare the occurrence rate of GBS reported to VAERS with the average GBS rate occurring in the general population, from 1994 to 2007. Patients were identified from the Nationwide Inpatient Sample database using the International Classification of Disease, 9th Revision, Clinical Modification code for a primary diagnosis of GBS (357.00). Annual census data of the population of participating states were also employed to calculate annual hospital discharge rates. The incidence of GBS in the general population was estimated based on a literature review. The medical literature was searched via MEDLINE, BIOSIS, and the Cochrane databases for pertinent publications from 1966 to 2010, using the key words previously mentioned. The weekly incidence of GBS was derived from the annual incidence and based on events that occurred 2-6 weeks after vaccination.

The total number of reported neurological events, life-threatening events, emergency department visits, hospitalizations, and disability, per 100,000 H1N1 and seasonal influenza vaccinated subjects, were estimated using the VAERS database.

\section{Results}

Sixty-two cases of GBS associated with H1N1 influenza vaccination were reported in the VAERS for the calendar year 2009, 33 of which occurred in men. The mean age ( \pm standard deviation) of these patients was $46.5 \pm$ 22.4 years. Miller-Fisher syndrome was the diagnosis in 6 cases (10\%). All reported cases of GBS occurred after administration of the inactivated $\mathrm{H} 1 \mathrm{~N} 1$ vaccine. In $6 \mathrm{GBS}$ cases (10\%), an antecedent illness within 4 weeks after vaccination was reported. In 3 of these cases, the preceding illness occurred within 2 weeks after vaccination, whereas the temporal relationship was not reported in the other 3 cases. GBS occurred in 54 subjects after administration of the $\mathrm{H} 1 \mathrm{~N} 1$ vaccine alone, in 5 cases after $\mathrm{H} 1 \mathrm{~N} 1$ 


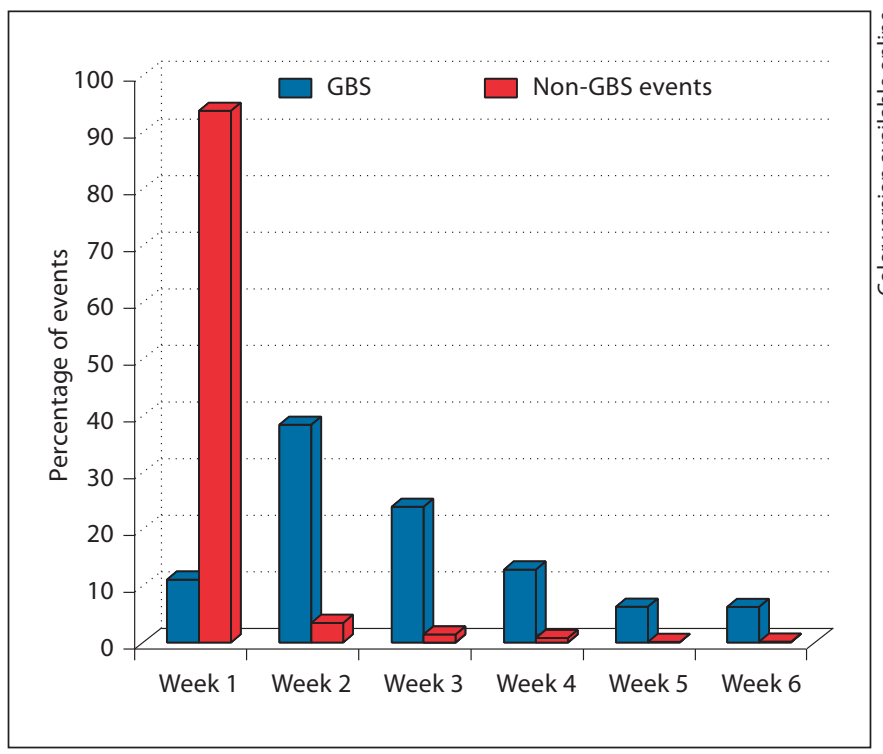

Fig. 1. Distribution of GBS and non-GBS events following the H1N1 vaccination in 2009.

vaccination combined with the seasonal influenza vaccine, and in 3 cases after $\mathrm{H} 1 \mathrm{~N} 1$ vaccination combined with other vaccines. All cases of GBS, except 2, were reported within 6 weeks after vaccination. The distribution of occurrence of GBS within the first 6 weeks showed a peak occurrence within the first 2 weeks after vaccination (fig. 1). Thirty-one patients (50\%) developed GBS within 2 weeks of vaccination (fig. 1). The probability of observing a distribution over the 6 weeks with at least this degree of asymmetry purely by chance was exceedingly low $(\mathrm{p}<0.0002)$. Two cases of disability and 1 death were reported due to $\mathrm{H} 1 \mathrm{~N} 1$ vaccine-associated GBS. The median interval between vaccination and GBS cases after H1N1 vaccination (14.5 days) was significantly longer than reported cases of non-GBS adverse events (1 day; $p<0.005)$. Of note, $94 \%$ of non-GBS adverse reactions after the H1N1 vaccine occurred within the first week after vaccination (fig. 1), with $74 \%$ of these events occurring in the first $24 \mathrm{~h}$.

In the year 2009, 57 patients were diagnosed with GBS after vaccination with the seasonal influenza vaccine. The onset of GBS was noted within 6 weeks after vaccination in 51 patients $(90 \%)$, most occurring within 2 weeks of vaccination ( $n=25,44 \%)$. In the case of 2 subjects ( $4 \%)$, the date of symptom onset was not reported. The median intervals between vaccination and GBS cases after the H1N1 vaccine (14.5 days), and after the seasonal influ-

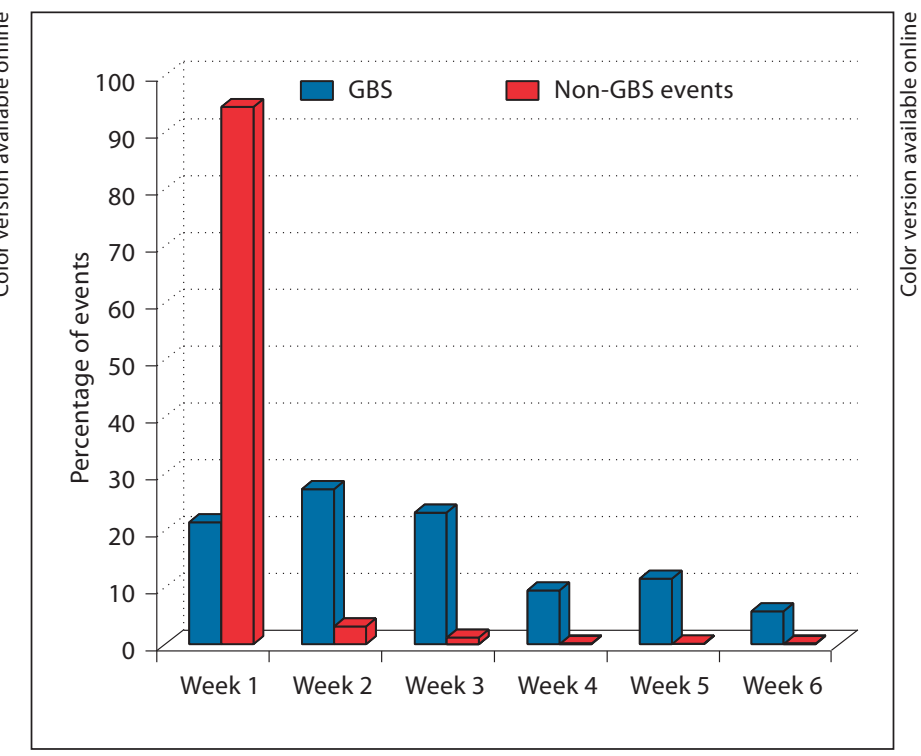

Fig. 2. Distribution of GBS and non-GBS events following the seasonal influenza vaccination in 2009.

enza vaccine (15 days) were similar. The median interval for onset of GBS cases after the seasonal influenza vaccine (15 days) was significantly longer than for reported cases of non-GBS adverse events (1 day; $\mathrm{p}<0.005)$. Most non-GBS adverse reactions (94.7\%) associated with the seasonal influenza vaccine occurred within the first week after vaccination (fig. 2), 76.81\% of which occurred within the first $24 \mathrm{~h}$.

Approximately 99.3 million doses of the H1N1 influenza vaccine were administered in the United States in 2009. Thus, the estimated annual reporting rate of GBS after vaccination with the $\mathrm{H} 1 \mathrm{~N} 1$ vaccine was approximately 6.2 per 10 million vaccinated subjects. The average weekly reporting rate of GBS within the first 6 weeks after $\mathrm{H} 1 \mathrm{~N} 1$ vaccination was 1.01 events per week, per 10 million subjects, whereas the average weekly reporting rate within the first 2 weeks after $\mathrm{H} 1 \mathrm{~N} 1$ vaccination was 1.56 events per week, per 10 million subjects. Data from the National Health Interview Survey, between 2000 and 2008 , indicate that an average of 86.4 million individuals are vaccinated with the seasonal influenza vaccine annually [19]. Given that 57 patients were diagnosed with GBS in 2009 after vaccination with the seasonal flu vaccine, the occurrence rate is 6.6 cases per 10 million vaccinations.

In the general population, the annual incidence of GBS is reported to be in the range of 34-134 cases per 10 mil- 


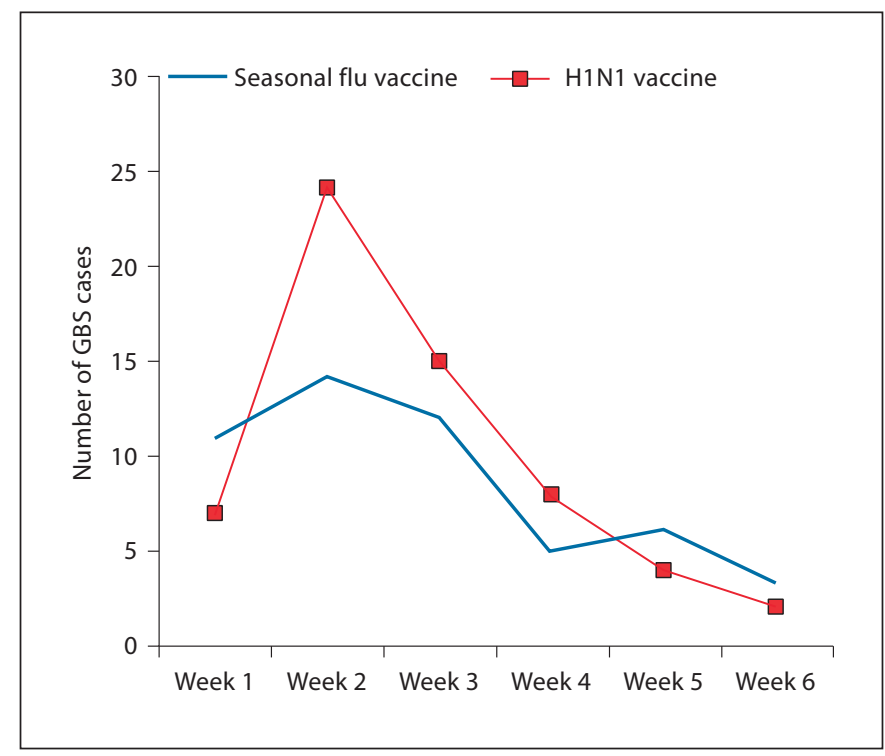

Fig. 3. Distribution of GBS after the seasonal influenza and H1N1 vaccinations in the United States in 2009.

Table 1. Events reported after H1N1 influenza vaccine and seasonal influenza vaccine administration (CDC/FDA Vaccine Adverse Event Reporting System, 2009)

\begin{tabular}{lll}
\hline \multirow{2}{*}{ Events } & \multicolumn{2}{l}{ Subjects vaccinated with } \\
\cline { 2 - 3 } & $\begin{array}{l}\text { the H1N1 } \\
\text { vaccine }\end{array}$ & $\begin{array}{l}\text { the seasonal } \\
\text { influenza } \\
\text { vaccine }\end{array}$ \\
& & \\
\hline Estimated number of vaccinated subjects & $99,366,920$ & $53,708,996$ \\
Total reports of significant events & 8,456 & 6,869 \\
Per $10^{5}$ subjects & 8.51 & 12.789 \\
Life-threatening events & 138 & 128 \\
Per $10^{5}$ subjects & 0.1389 & 0.238 \\
Emergency room visits & 2,956 & 2,317 \\
Per $10^{5}$ subjects & 2.975 & 4.31 \\
Hospitalizations & 523 & 490 \\
Per $10^{5}$ subjects & 0.526 & 0.912 \\
Disability & 63 & 89 \\
Per $10^{5}$ subjects & 0.0634 & 0.166 \\
Death rate & 51 & 45 \\
Per $10^{5}$ subjects & 0.0513 & 0.0838 \\
GBS cases & 62 & 57 \\
\hline
\end{tabular}

lion [20]. This corresponds to an average incidence of $0.65-2.57$ cases per week, per 10 million persons. However, when Nationwide Inpatient Sample data were analyzed, the average incidence of GBS between 1994 and 2007 was calculated to have been between 200 and 259 cases per 10 million, corresponding to $3.84-5$ cases per week, per 10 million persons. Therefore, the reporting rate of GBS after vaccination, with either the seasonal influenza or H1N1 vaccine, is within the expected ranges for the general population. For both vaccines, the distribution of GBS in the first 6 weeks after vaccination was unbalanced, with a peak occurrence rate in the first 2 weeks (fig. 3).

In 2009, there were no significant differences in the VAERS reports of life-threatening events, emergency department visits, hospitalizations, and disability between the $\mathrm{H} 1 \mathrm{~N} 1$ and seasonal influenza vaccines (table 1).

\section{Discussion}

Although previous clinical trials reported that 10 $49 \%$ of subjects vaccinated with the H1N1 influenza vaccine developed mild adverse reactions, the occurrence of GBS was not investigated $[7,21]$. We found a similar rate of GBS occurrence after administration of the influenza A (H1N1) vaccine in 2009 as that observed after the seasonal influenza vaccine. The temporal distribution of GBS occurrence following H1N1 influenza vaccination was similar to GBS following seasonal influenza vaccination, with peak occurrence within the first 2 weeks after vaccination, followed by a continuous decline in the 3 - to 6-week period (fig. 1, 3). A similar temporal distribution for GBS occurrence following the seasonal influenza vaccine was reported by Lasky et al. [15] and Haber et al. [16]. The greatest relative risk of GBS onset after a respiratory infection, a potential triggering factor, was also reported in the first 2 weeks after infection [22]. The temporal distribution of GBS after H1N1 vaccination supports a potential causal role for the vaccine. The low prevalence of viral infection or other illnesses in the 4 weeks that preceded GBS onset (10\%), unlike that which usually occurs in non-vaccine-related GBS cases (61.8\%), also supports a causal relationship $[13,16]$. Molecular mimicry and other immune system stimulation mechanisms are plausible explanations mediating the occurrence of GBS after H1N1 influenza vaccination. The high antigenicity of the vaccine, or one of its components, may trigger GBS in genetically predisposed individuals in a manner similar to other vaccines $[23,24]$.

This study has limitations that need to be considered when interpreting the results. Case ascertainment was based on VAERS reports, a passive surveillance system that is subject to underreporting, differential reporting, ascertainment bias, and variability in report quality and 
completeness [16]. The actual reporting rate of GBS following vaccination may be higher because the reporting system is voluntary and therefore, it is possible that some GBS events were not reported to the VAERS. Inclusion of non-GBS cases entered as GBS in VAERS following vaccination may result in overestimation, but this concern is reduced because of an independent review of the data by the Center for Biologics Evaluation and Research, and by a certified neuromuscular specialist in our study. In a study focusing on validation of GBS reports in the VAERS database, Haber et al. [16] conducted an active follow-up of GBS reports from 1994 through 2003 and found that $82 \%$ of the reported cases were true GBS events. Therefore, the accuracy of diagnosis even in the absence of independent review appears to be high.

Occurrence of GBS following H1N1 influenza vaccination may cause public concern and reluctance to comply with CDC recommendations, which may in turn influence public policy on vaccination, leading to widespread underutilization of vaccines $[25,26]$. Our results need to be considered in light of the overwhelming benefit of H1N1 vaccinations in reducing viral illness-associated morbidity and mortality [27]. Although our study suggests that GBS events may be potentially triggered by the $\mathrm{H} 1 \mathrm{~N} 1$ influenza vaccine, the incidence is very low. We thus interpret these results as being supportive of the current guidelines for vaccination, especially when weighing the risk/benefit ratio. Our results serve to increase awareness among health care providers involved in post-vaccination care to ensure early identification of GBS cases and institution of immunotherapy for its treatment. As such, physicians should explain the favorable risk/benefit ratio to their patients in order to improve adherence to public health vaccination guidelines.

\section{Conclusion}

To our knowledge, this is one of the first reports to provide data on GBS occurrence after H1N1 influenza vaccination, the temporal distribution, and outcomes. This observation warrants early recognition and treatment through active surveillance for GBS after H1N1 influenza vaccination, but should not obviate the overwhelming benefit of such vaccination.

\section{References}

1 Pandemic (H1N1) 2009 - update 90. http:// www.Who.Int/csr/don/2010_03_05/en/index.html (accessed March 14, 2010).

2 Swine influenza A (H1N1) infection in two children - Southern California, MarchApril 2009. MMWR Morb Mortal Wkly Rep 2009;58:400-402.

- 3 Perez-Padilla R, de la Rosa-Zamboni D, Ponce de Leon S, Hernandez M, QuinonesFalconi F, Bautista E, Ramirez-Venegas A, Rojas-Serrano J, Ormsby CE, Corrales A, Higuera A, Mondragon E, Cordova-Villalobos JA: Pneumonia and respiratory failure from swine-origin influenza A (H1N1) in Mexico. N Engl J Med 2009;361:680-689.

4 Updated CDC estimates of $2009 \mathrm{H} 1 \mathrm{~N} 1 \mathrm{influ}$ enza cases, hospitalizations and deaths in the United States, April 2009 - April 10, 2010. http://www.Cdc.Gov/h1n 1 flu/estimates_2009_h1n1.htm (accessed on August $4,2011)$.

5 Use of influenza A (H1N1) 2009 monovalent vaccine recommendations of the advisory committee on immunization practices (ACIP), 2009. http://www.Cdc.Gov/ $\mathrm{mmwr} /$ preview/mmwrhtml/rr5810a $1 . \mathrm{htm}$ (accessed March 14, 2010).

-6 Jain R, Goldman RD: Novel influenza A (H1N1): clinical presentation, diagnosis, and management. Pediatr Emerg Care 2009;25: 791-796.
7 Liang XF, Wang HQ, Wang JZ, Fang HH, Wu J, Zhu FC, Li RC, Xia SL, Zhao YL, Li FJ, Yan SH, Yin WD, An K, Feng DJ, Cui XL, Qi FC, Ju CJ, Zhang YH, Guo ZJ, Chen PY, Chen Z, Yan KM, Wang Y: Safety and immunogenicity of 2009 pandemic influenza A H1N1 vaccines in China: a multicentre, double-blind, randomised, placebo-controlled trial. Lancet 2010;375:56-66.

8 Vajo Z, Tamas F, Sinka L, Jankovics I: Safety and immunogenicity of a 2009 pandemic influenza A H1N1 vaccine when administered alone or simultaneously with the seasonal influenza vaccine for the 2009-10 influenza season: a multicentre, randomised controlled trial. Lancet 2010;375:49-55.

$\checkmark 9$ Souayah N, Michas-Martin PA, Nasar A, Krivitskaya N, Yacoub HA, Khan H, Qureshi AI: Guillain-Barré syndrome after Gardasil vaccination: data from Vaccine Adverse Event Reporting System 2006-2009. Vaccine 2011;29:886-889.

10 Souayah N, Nasar A, Suri MF, Qureshi AI: Guillain-Barré syndrome after vaccination in United States: data from the Centers for Disease Control and Prevention/Food and Drug Administration Vaccine Adverse Event Reporting System (1990-2005). J Clin Neuromuscul Dis 2009;11:1-6.
11 Souayah N, Nasar A, Suri MF, Qureshi AI: Guillain-Barré syndrome after vaccination in United States: a report from the CDC/FDA Vaccine Adverse Event Reporting System. Vaccine 2007;25:5253-5255.

12 Moon JS, Souayah N: Guillain-Barré syndrome triggered by influenza vaccination in a recipient of liver transplant on FK506. Liver Transpl 2006;12:1537-1539.

13 Schonberger LB, Bregman DJ, SullivanBolyai JZ, Keenlyside RA, Ziegler DW, Retailliau HF, Eddins DL, Bryan JA: GuillainBarré syndrome following vaccination in the National Influenza Immunization program, United States, 1976-1977. Am J Epidemiol 1979;110:105-123.

-14 Safranek TJ, Lawrence DN, Kurland LT, Culver DH, Wiederholt WC, Hayner NS, Osterholm MT, O’Brien P, Hughes JM: Reassessment of the association between GuillainBarré syndrome and receipt of swine influenza vaccine in 1976-1977: results of a two-state study. Expert Neurology Group. Am J Epidemiol 1991;133:940-951.

15 Lasky T, Terracciano GJ, Magder L, Koski CL, Ballesteros M, Nash D, Clark S, Haber P, Stolley PD, Schonberger LB, Chen RT: The Guillain-Barré syndrome and the 1992-1993 and 1993-1994 influenza vaccines. N Engl J Med 1998;339:1797-1802. 
16 Haber P, DeStefano F, Angulo FJ, Iskander J, Shadomy SV, Weintraub E, Chen RT: Guillain-Barré syndrome following influenza vaccination. JAMA 2004;292:2478-2481.

17 Chumney EC, Biddle AK, Simpson KN, Weinberger M, Magruder KM, Zelman WN The effect of cost construction based on either DRG or ICD-9 codes or risk group stratification on the resulting cost-effectiveness ratios. Pharmacoeconomics 2004;22:12091216.

18 Simpson L, Owens PL, Zodet MW, Chevarley FM, Dougherty D, Elixhauser A, McCormick MC: Health care for children and youth in the United States: annual report on patterns of coverage, utilization, quality, and expenditures by income. Ambul Pediatr 2005;5:6-44.
19 CDC: Immunization coverage in the US. http://www.Cdc.Gov/vaccines/stats-surv/ imz-coverage.htm (accessed March 23, 2010).

20 McGrogan A, Madle GC, Seaman HE, de Vries CS: The epidemiology of Guillain-Barré syndrome worldwide. A systematic literature review. Neuroepidemiology 2009;32: 150-163.

21 Plennevaux E, Sheldon E, Blatter M, ReevesHoche MK, Denis M: Immune response after a single vaccination against 2009 influenza A H1N1 in USA: a preliminary report of two randomised controlled phase 2 trials. Lancet 2010;375:41-48.

22 Winer JB, Hughes RA, Anderson MJ, Jones DM, Kangro H, Watkins RP: A prospective study of acute idiopathic neuropathy. 2. Antecedent events. J Neurol Neurosurg Psychiatry 1988;51:613-618.
3 Shoenfeld Y, Aron-Maor A: Vaccination and autoimmunity-'vaccinosis': a dangerous liaison? J Autoimmun 2000;14:1-10.

24 Smith JF, Brownlow M, Brown M, Kowalski R, Esser MT, Ruiz W, Barr E, Brown DR, Bryan JT: Antibodies from women immunized with Gardasil cross-neutralize HPV 45 pseudovirions. Hum Vaccin 2007;3:109-115.

25 Patja A, Paunio M, Kinnunen E, Junttila O, Hovi T, Peltola H: Risk of Guillain-Barré syndrome after measles-mumps-rubella vaccination. J Pediatr 2001;138:250-254.

26 Deaths and hospitalizations related to 2009 pandemic influenza A (H1N1) - Greece, May 2009-February 2010. MMWR Morb Mortal Wkly Rep 2010;59:682-686.

27 Roush SW, Murphy TV: Historical comparisons of morbidity and mortality for vaccinepreventable diseases in the United States. JAMA 2007;298:2155-2163. 